\title{
Fields in the Language of String: Divergences and Renormalization
}

\author{
Charles B. Thorn*† \\ Institute for Fundamental Theory, Department of Physics, University of Florida, \\ Gainesville FL 32611 \\ E-mail: thhorn@phys.ufi.edu'
}

Abstract: After a brief introduction to the lightcone worldsheet formalism [1] for summing the planar diagrams of field theory, I explain how the $u v$ divergences of quantum field theory translate to the new language of string. It is shown through one loop that, at least for scalar cubic vertices, the counter-terms necessary for Poincaré invariance in space-time dimensions $D \leq 6$ are indeed local on the worldsheet. The extension to cover the case of gauge field vertices will be more complicated due to the extra divergences at $p^{+}=0$ in lightcone gauge.

\section{Introduction}

Two years ago Bardakci and I developed a new formalism [1] for mapping the sum of all planar diagrams [2] of a cubic scalar quantum field theory onto a two dimensional system defined on the worldsheet of lightcone string theory. Since then the formalism has been extended to cover Yang-Mills theory [3] and extended supersymmetric gauge theories with $\mathcal{N}=1,2,4[4]$.

Much of my talk to this workshop was devoted to a pedagogical explanation of the new worldsheet formalism. However, since this part of the talk was virtually the same as one given at the August 2003 lightcone meeting [5], I limit this introduction to a brief synopsis of the formulas needed to understand the new results reported here on how field theoretic divergences can be dealt with locally on the worldsheet. The worldsheet is based on light-cone parameters, an imaginary time $\tau=i x^{+}=i(t+z) / \sqrt{2}$ in the range $0 \leq \tau \leq T$, and a worldsheet spatial coordinate $0 \leq \sigma \leq p^{+}$chosen so that the $p^{+}$density is uniform $[6]$.

\footnotetext{
${ }^{*}$ Speaker.

${ }^{\dagger}$ Supported in part by the Department of Energy under Grant No. DE-FG02-97ER-41029.
} 
The key to the worldsheet representation of an arbitrary planar diagram is that of a free scalar gluon propagator, $\theta(T) e^{-T\left(\boldsymbol{p}^{2}+\mu^{2}\right) / 2 p^{+}}$in lightcone variables. It is based on the remarkable identity [1]

$$
\exp \left\{-\frac{T \boldsymbol{p}^{2}}{2 p^{+}}\right\}=\int D c D b D \boldsymbol{q} \exp \left\{-\int d \tau \int_{0}^{p^{+}} d \sigma\left[\frac{1}{2} \boldsymbol{q}^{\prime 2}-\boldsymbol{b}^{\prime} \boldsymbol{c}^{\prime}\right]\right\}
$$

where Dirichlet boundary conditions are imposed $\dot{\boldsymbol{q}}=0$ at $\sigma=0, p^{+}$, and also $\boldsymbol{q}\left(p^{+}\right)-$ $\boldsymbol{q}(0)=\boldsymbol{p}$. The Grassmann variables, with boundary conditions $\boldsymbol{b}=\boldsymbol{c}=0$ at $\sigma=0, p^{+}$ assure the correct measure and ' is shorthand for $\partial / \partial \sigma$. The absence of time derivatives in $S$ reflects the topological nature of the free worldsheet dynamics. Note that in $D=d+2$ space-time dimensions we have all together $d / 2$ sets of $\boldsymbol{b}, \boldsymbol{c}$ ghost pairs, denoted by boldfaced letters.

We give rigorous meaning to this formula using a worldsheet lattice [7]: $\tau=k a$ and $\sigma=l m$ with $T=N a$ and $p^{+}=M m$. The limit of a continuous worldsheet is equivalent to the double limit $M, N \rightarrow \infty$ with $N / M=\left(T / p^{+}\right)(m / a)$ fixed. Then defining

$$
\begin{aligned}
S \equiv \frac{a}{2 m} \sum_{j=1}^{N} \sum_{i=0}^{M-1}\left(\boldsymbol{q}_{i+1}^{j}-\boldsymbol{q}_{i}^{j}\right)^{2} & \\
& -\frac{a}{m} \sum_{j=1}^{N}\left[(1+\rho) \boldsymbol{b}_{1}^{j} \boldsymbol{c}_{1}^{j}+\boldsymbol{b}_{M-1}^{j} \boldsymbol{c}_{M-1}^{j}+\sum_{i=1}^{M-2}\left(\boldsymbol{b}_{i+1}^{j}-\boldsymbol{b}_{i}^{j}\right)\left(\boldsymbol{c}_{i+1}^{j}-\boldsymbol{c}_{i}^{j}\right)\right]
\end{aligned}
$$

the master formula on the worldsheet lattice is [1]

$$
\left(1-\frac{a \mu^{2}}{d m M}\right)^{d N / 2} \exp \left\{-\frac{N a \boldsymbol{p}^{2}}{2 m M}\right\}=\frac{1}{(1+\rho)^{d N / 2}} \int \prod_{j=1}^{N} \prod_{i=1}^{M-1} \frac{d \boldsymbol{c}_{i}^{j} d \boldsymbol{b}_{i}^{j}}{2 \pi} d \boldsymbol{q}_{i}^{j} e^{-S},
$$

with boundary conditions $\boldsymbol{q}_{0}^{j}=\boldsymbol{q}_{0}, \boldsymbol{q}_{M}^{j}=\boldsymbol{q}_{0}+\boldsymbol{p}, \boldsymbol{b}_{0, M}^{j}=\boldsymbol{c}_{0, M}^{j}=0$. The parameter $\rho=\mu^{2} a /\left(d m-\mu^{2} a\right)$ provides a mass $\mu$ for the gluon in the continuum limit. The prefactor on the right can be associated with the left boundary.

The worldsheet lattice provides a template for summing all planar diagrams in the cubic theory. We can use the ratio of lattice constants $m / a$, with units of energy/time, to define a dimensionless coupling

$$
\hat{g}^{2} \equiv \frac{g^{2}}{64 \pi^{3}}\left(\frac{m}{2 \pi a}\right)^{(d-4) / 2} .
$$

The worldsheet for the general planar diagram has an arbitrary number of vertical solid lines marking the location of the internal boundaries corresponding to loops. Each interior link $j, j-1$ of a solid line at spatial location $k$ requires a factor of $\delta\left(\boldsymbol{q}_{k}^{j}-\boldsymbol{q}_{k}^{j-1}\right)$. To supply such factors, assign an Ising spin $s_{k}^{j}= \pm 1$ to each site of the lattice. We assign +1 if the site $(k, j)$ is crossed by a vertical solid line, -1 otherwise. We also use the spin up projector $P_{k}^{j}=\left(1+s_{k}^{j}\right) / 2$. We implement the Dirichlet conditions on boundaries using the Gaussian representation of the delta function:

$$
\left(\frac{2 \pi m}{a}\right)^{d / 2} \delta\left(\boldsymbol{q}_{i}^{j}-\boldsymbol{q}_{i}^{j-1}\right)=\lim _{\epsilon \rightarrow 0} \frac{1}{\epsilon^{d / 2}} \exp \left\{-\frac{a}{2 m \epsilon}\left(\boldsymbol{q}_{i}^{j}-\boldsymbol{q}_{i}^{j-1}\right)^{2}\right\}
$$


We keep $\epsilon$ finite until the end of the calculation. Using this device, our formula for the sum of planar diagrams is:

$$
\begin{aligned}
& T_{f i}=\lim _{\epsilon \rightarrow 0} \sum_{s_{i}^{j}= \pm 1} \int D c D b D \boldsymbol{q} \exp \left\{\ln \hat{g} \sum_{i j} \frac{1-s_{i}^{j} s_{i}^{j-1}}{2}-\frac{d}{2} \ln (1+\rho) \sum_{i, j} P_{i}^{j}\right\} \\
& \exp \left\{-\frac{a}{2 m} \sum_{i, j}\left(\boldsymbol{q}_{i+1}^{j}-\boldsymbol{q}_{i}^{j}\right)^{2}-\frac{a}{2 m \epsilon} \sum_{i, j} P_{i}^{j} P_{i}^{j-1}\left(\boldsymbol{q}_{i}^{j}-\boldsymbol{q}_{i}^{j-1}\right)^{2}\right\} \\
& \exp \left\{\frac{a}{m} \sum_{i, j}\left[A_{i j} \boldsymbol{b}_{i}^{j} \boldsymbol{c}_{i}^{j}-B_{i j} b_{i}^{j} c_{i}^{j}+C_{i j}\left(\boldsymbol{b}_{i+1}^{j}-\boldsymbol{b}_{i}^{j}\right)\left(\boldsymbol{c}_{i+1}^{j}-\boldsymbol{c}_{i}^{j}\right)-D_{i j}\left(b_{i+1}^{j}-b_{i}^{j}\right)\left(c_{i+1}^{j}-c_{i}^{j}\right)\right]\right\} \\
& A_{i j}=\frac{1}{\epsilon} P_{i}^{j} P_{i}^{j-1}+P_{i}^{j+1} P_{i}^{j}-P_{i}^{j-1} P_{i}^{j} P_{i}^{j+1}+\left(1-P_{i}^{j}\right)\left(P_{i+1}^{j}+P_{i-1}^{j}\right)+\rho\left(1-P_{i}^{j}\right) P_{i-1}^{j-1} P_{i-1}^{j} \\
& B_{i j}=\left(1-P_{i}^{j}\right)\left(P_{i+1}^{j} P_{i+1}^{j+1}\left(1-P_{i+1}^{j-1}\right)+P_{i-1}^{j} P_{i-1}^{j+1}\left(1-P_{i-1}^{j-1}\right)+P_{i}^{j-1} P_{i}^{j-2} P_{i+1}^{j}\right) \\
& C_{i j}=\left(1-P_{i}^{j}\right)\left(1-P_{i+1}^{j}\right) \\
& D_{i j}=\left(1-P_{i}^{j}\right)\left(1-P_{i+1}^{j}\right) P_{i}^{j-1} P_{i}^{j-2}
\end{aligned}
$$

The first exponent in (1) stroyed. The second exponent includes the action $S_{q}$ for the free propagator together with the exponent in the Gaussian representation of the delta function that enforces Dirichlet boundary conditions on the solid lines. The first term of the third exponent incorporates the $\epsilon$ dependent prefactor in the representation of the delta function as a term in the ghost Lagrangian. The remaining terms contain $S_{g}$ together with strategically placed spin projectors that arrange the proper boundary conditions on the Grassmann variables and supply appropriate $1 / p^{+}$factors needed at the beginning or end of solid lines to ensure Lorentz invariance.

\section{Self Energy for $\Phi^{3}$}

The worldsheet lattice for the one loop self energy is drawn in Fig. ii.i. The solid line segment in the middle of the diagram is the internal boundary that separates the two propagators of the two gluon intermediate state. We take $\epsilon=0$ from the beginning, so exact Dirichlet boundary conditions are imposed. For this single diagram, the worldsheet path integral, (1).6i) with $\epsilon \rightarrow 0$ and $\boldsymbol{q}_{l}^{j}=\boldsymbol{q}$ fixed on the internal boundary, immediately reduces to the usual light-cone Feynman rules. Then we evaluate the $\boldsymbol{q}$ integration and take $N$ large:

$$
\begin{aligned}
T_{\mathrm{SE}} & =\left(\frac{a}{2 \pi m}\right)^{d / 2} \sum_{k_{0}, k, l} \frac{\hat{g}^{2}}{M l(M-l)} \int d \boldsymbol{q} \\
& \quad \exp \left\{\frac{-N a\left(\boldsymbol{p}^{2}+\mu^{2}\right)}{2 m M}-\frac{k a M}{2 m l(M-l)}\left(\boldsymbol{q}^{2}+\mu^{2} \frac{M^{2}-l(M-l)}{M^{2}}\right)\right\} \\
& \sim N \frac{\hat{g}^{2}}{M^{2}} \sum_{k, l}\left[\frac{l(M-l)}{M}\right]^{\frac{d}{2}-1} \frac{1}{k^{d / 2}} \exp \left\{-\frac{k a \mu^{2}}{2 m}\left(\frac{M}{l(M-l)}-\frac{1}{M}\right)\right\}
\end{aligned}
$$




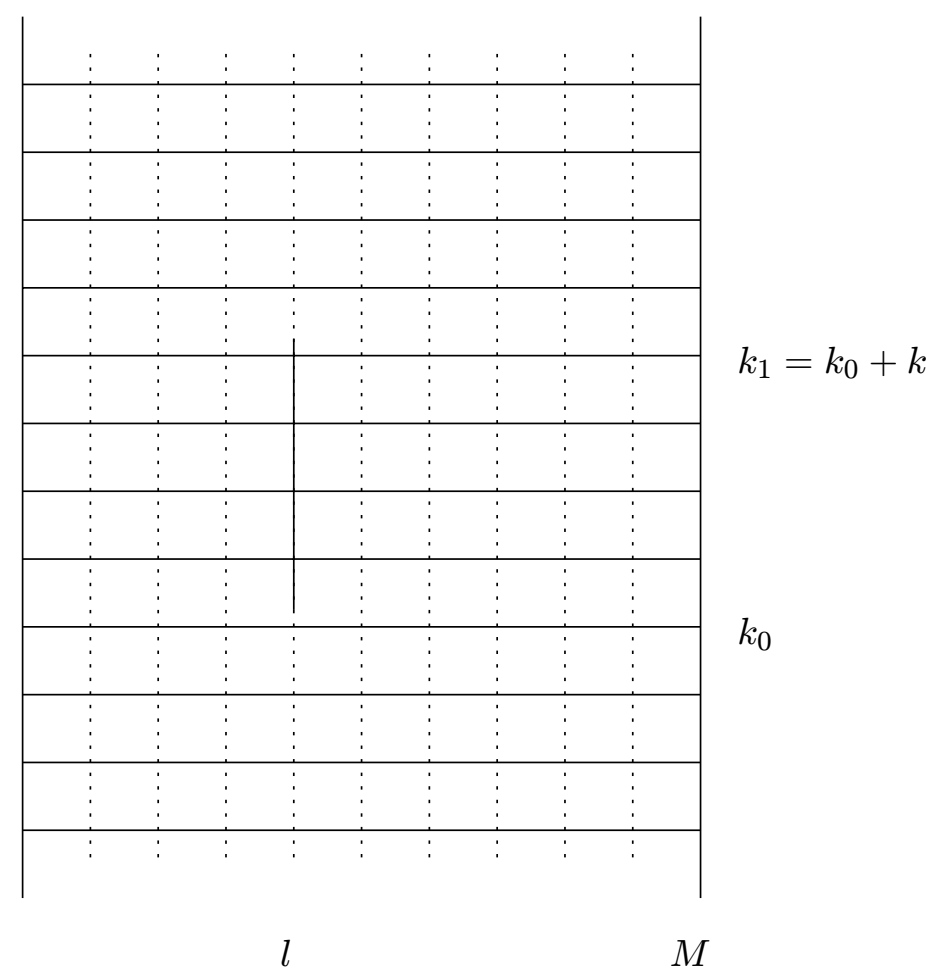

Figure 1: Worldsheet lattice for self-energy

The factor of $N=T / a$ simply reflects time translational invariance, and leads to the interpretation of its coefficient as $-a$ times a shift in energy, $-a \delta p^{-}=-a \delta \mu^{2} / 2 m M$. Thus we have

$$
\delta \mu^{2}=-\frac{2 m \hat{g}^{2}}{a M} \sum_{k, l}\left[\frac{l(M-l)}{M}\right]^{\frac{d}{2}-1} \frac{1}{k^{d / 2}} \exp \left\{-\frac{k a \mu^{2}}{2 m}\left(\frac{M}{l(M-l)}-\frac{1}{M}\right)\right\} .
$$

We see that the worldsheet lattice has provided a cutoff for the usual field theoretic ultraviolet divergences. The removal of this cutoff in this quantity is simply taking the limit $M \rightarrow \infty$, which is the limit of a continuous worldsheet. More generally, for the finite time transition amplitude, the continuum limit is $M, N \rightarrow \infty$, with $N a / M m=T / p^{+}$fixed.

We first observe that this limit is well defined as long as $d<2$. The $k, l$ sums go to integrals over continuous variables $t=\left(k a \mu^{2} / 2 m\right)(M / l(M-l)-1 / M)$ and $x=l / M$ :

$$
\sum_{k} \frac{1}{k^{d / 2}} \rightarrow \int \frac{d t}{t^{d / 2}}\left[\frac{a \mu^{2}}{2 m}\left(\frac{M}{l(M-l)}-\frac{1}{M}\right)\right]^{(d-2) / 2}, \quad \frac{1}{M} \sum_{l} \rightarrow \int_{0}^{1} d x
$$

Then we find

$$
\delta \mu^{2} \rightarrow-\mu^{2} \hat{g}^{2}\left[\frac{a \mu^{2}}{2 m}\right]^{(d-4) / 2} \Gamma\left(1-\frac{d}{2}\right) \int_{0}^{1} d x(1-x(1-x))^{(d-2) / 2}+O\left(M^{(d-2) / 2}\right) .
$$

Order by order in perturbation theory, one could invoke dimensional regularization, which defines divergent quantities as the continuation in dimension from a region where they are 
finite. Then we would conclude from this formula that the mass shift is Lorentz invariant. Divergences in mass shift would appear as Lorentz invariant poles at integer $d \geq 2$, and they could be covariantly absorbed in the input mass parameter $\mu$.

However, dimensional regularization is pretty useless for non-perturbative numerical work, because, in the presence of an ultraviolet cutoff (necessary for digitizing the problem), calculations at general $d$ contain non-covariant artifacts, which are negligible only in sufficiently small dimensions. It is essential in dimensional regularization to take the continuum limit with $d$ in a range where the quantity is finite before continuing to the physical dimension, a procedure that is impossible on a computer.

Indeed, the cutoff provided by the worldsheet lattice introduces non-covariant artifacts simply because the cutoff $M=p^{+} / m$ is a component of momentum so all divergent terms introduce frame dependence. We can see why this happens by noting that the corrections to the continuum limit for $d<2$ are of $O\left(M^{(d-2) / 2}\right)$ and obviously non-covariant. As $d \rightarrow 2$ these terms fall off more and more slowly eventually becoming comparable to the Lorentz invariant term and then for $d>2$ dominating it. Thus the worldsheet lattice by itself will consistently produce Lorentz invariant results only when divergences are completely absent, i.e. for $d<2$.

If we want to numerically analyze the system for $d \geq 2$ without introducing an additional $u v$ cutoff, the worldsheet lattice must be supplemented with explicit counter-terms that remove the Lorentz violating artifacts introduced by divergences. For example, at $d=2$ a logarithmic divergence in the self energy appears as $\ln M=\ln (1 / m)+\ln p^{+}$, and the non-covariance is actually in the finite part. There is the distinct possibility that the necessary counter-terms are not local on the worldsheet, though it is relatively easy to find local counter-terms that fix the problems in the self energy at least for $d=2,4$.

To see this, take the interesting asymptotically free case of $\operatorname{Tr} \Phi^{3}$ scalar field theory in $D=6$ space-time dimensions $(d=4)$. Then in the self-energy one encounters the $\delta \rightarrow 0$ limit of the quantity

$$
\sum_{k=1}^{\infty} \frac{e^{-k \delta}}{k^{2}} \rightarrow \frac{\pi^{2}}{6}+\delta \ln \delta+O\left(\delta^{2}\right),
$$

and one finds for the mass shift

$$
\begin{gathered}
\delta \mu^{2} \rightarrow-\frac{2 m}{a} \hat{g}^{2} \sum_{l} x(1-x)\left[\frac{\pi^{2}}{6}+\delta_{l} \ln \delta_{l}\right] \\
x=\frac{l}{M}, \quad \delta_{l}=\frac{a \mu^{2}}{2 M m} \frac{1-x(1-x)}{x(1-x)},
\end{gathered}
$$

and then, as $M \rightarrow \infty$, the behavior

$$
\delta \mu^{2} \rightarrow C_{1} M+C_{0}^{\prime} \ln M+C_{0}
$$

Clearly the $C_{1}, C_{0}^{\prime}$ terms are non-covariant, and they must be removed by counter-terms. This can be done locally on the worldsheet. First note that the $C_{1}$ term can be canceled by a constant energy shift, which can be interpreted as a worldsheet boundary term (i.e. 
a perimeter cosmological constant). Then one can devise an isolated up spin with ghost insertions to contribute a counter-term $\sum_{l} \frac{1}{l} \sim \ln M$ to cancel $C_{0}^{\prime}$. The remaining $C_{0}$ term is just a covariant mass shift. It is not at all clear, however, that the counter-terms needed in vertex loop diagrams can also be prescribed locally on the worldsheet. One of the longstanding drawbacks of standard lightcone gauge perturbation theory is the need for counter-terms that are not polynomials in the $p^{+}$'s, and there is no simple a priori principle for specifying them. It is possible that worldsheet locality provides such a principle. If true, this would give an a priori justification to apply the logic of string theory to help define and solve large $N_{c}$ QCD. Bardakci [10] has stressed that $M^{+-}$boost invariance is precisely worldsheet scale invariance. Perhaps the remaining $M^{k-}$ Lorentz invariance is the underlying physical reason for worldsheet conformal invariance.

In the following we take an alternative more systematic approach, based on observations Glazek has made about controlling Lorentz invariance in the light-cone formalism [11]. The troubles outlined above can be traced to the way the worldsheet lattice cuts off the transverse momentum integrals, $\Lambda_{\perp} \propto M m / a$, so the continuous worldsheet $M \rightarrow \infty$ has no $u v$ cutoff. This can be cured by introducing an $M$ independent cutoff on transverse momentum which is held fixed as $M \rightarrow \infty$. The theoretical drawback is that it violates invariance under a Galilei subgroup of the Lorentz group. However we shall find that the problem of restoring this invariance is not severe.

The simplest way to implement an $M$ independent $u v$ cutoff in transverse target space is to include a factor $e^{-\delta \boldsymbol{q}_{k}^{j 2} / 2}$ in the world sheet path integrand whenever $(k, j)$ marks the beginning of a solid line. ${ }^{1}$ That is, we add terms $\delta\left(1-P_{k}^{j-1}\right) P_{k}^{j} \boldsymbol{q}_{k}^{j 2} / 2$ to the worldsheet action. These terms obviously violate Galilei invariance (a part of Lorentz invariance), and we must be careful that the invariance is restored after the limit $\delta \rightarrow 0$.

Let us now redo the self energy calculation with $\delta \neq 0$.

$$
\begin{aligned}
\delta \mu^{2}= & -\frac{2 m \hat{g}^{2}}{a} \sum_{k, l} \frac{1}{l(M-l)} \frac{1}{(k M / l(M-l)+m \delta / a)^{d / 2}} \\
& \exp \left\{-\frac{k a \mu^{2}}{2 m}\left(\frac{M}{l(M-l)}-\frac{1}{M}\right)-\frac{k a \delta}{2 M}\left[\frac{\left(\boldsymbol{p}_{1} l+\boldsymbol{p}_{0}(M-l)\right)^{2}}{k a M+\delta l(M-l) m}\right]\right\} .
\end{aligned}
$$

Now we can safely take the continuum limit $M \rightarrow \infty$. Define $T=k a M / l(M-l) m$ and $x=l / M$, we find

$$
\begin{aligned}
\delta \mu^{2}= & -2 \hat{g}^{2}\left(\frac{m}{a}\right)^{2-d / 2} \int_{0}^{1} d x \int_{0}^{\infty} d T \frac{1}{(T+\delta)^{d / 2}} \\
& \exp \left\{-\frac{T \mu^{2}}{2}(1-x(1-x))-T \frac{\delta}{2}\left[\frac{\left(x \boldsymbol{p}_{1}+(1-x) \boldsymbol{p}_{0}\right)^{2}}{T+\delta}\right]\right\} .
\end{aligned}
$$

The explicit dependence on the boundary values of $\boldsymbol{q}$ reflects the violation of Galilei boost invariance introduced by the cutoff $\delta$ : this is the price paid for regaining manifest longitudinal Lorentz boost invariance. Inspection of the formula shows that these Galilei boost

\footnotetext{
${ }^{1}$ This device was first used in [8] to facilitate a mean field approximation [9] on the worldsheet.
} 
violations will disappear for $\delta \rightarrow 0$ as long as $d<4$, i.e. in less that 6 space-time dimensions. In this case, the divergences can be absorbed in a shift of $\mu^{2}$ consistently with Lorentz invariance and with no counter-terms.

We do want to study the 6 dimensional case, so we can't quite escape the need for counter-terms. To study this issue for the self energy, we set $d=4$ and analyze the $\delta \rightarrow 0$ behavior of the mass shift.

$$
\begin{aligned}
\delta \mu^{2} & =-2 \hat{g}^{2} \int_{0}^{1} d x e^{\delta(\alpha(x)-\beta(x))} \int_{\delta}^{\infty} d T \frac{1}{T^{2}} \exp \left\{-T \alpha(x)+\frac{\delta^{2}}{T} \beta(x)\right\} \\
& =-2 \hat{g}^{2} \int_{0}^{1} d x\left(\frac{1}{\delta}+\ln \delta-\frac{\beta(x)}{2}-\alpha^{2}(x) \int_{0}^{\infty} d T \ln T e^{-\alpha(x) T}\right)+O(\delta) \\
\alpha(x) & =\frac{\mu^{2}}{2}(1-x(1-x)) \\
\beta(x) & =\frac{1}{2}\left[(1-x) \boldsymbol{p}_{0}+x \boldsymbol{p}_{1}\right]^{2}
\end{aligned}
$$

In the $\delta \rightarrow 0$ limit the non-covariant artifact resides in the term $\hat{g}^{2} \int d x \beta(x)$, a finite positive contribution to $\delta \mu^{2}$. The corresponding contribution to the path integral is of course

$$
-T \delta \mu^{2} / 2 p^{+}=-(a N / 2 m M) \hat{g}^{2} \int d x \beta(x) .
$$

All of the divergences can be covariantly absorbed in a mass shift. But we still need to design a worldsheet local counter-term that removes this finite but non-covariant artifact.

To construct a suitable counter term we recall from [3] the generating formula for correlators of $\boldsymbol{q}_{i}^{j}$ on a fixed time slice $j$ of the worldsheet path integral representation of the free propagator:

$$
\begin{array}{r}
\left\langle\exp \left\{\sum_{i=1}^{M-1} J_{i} q_{i}\right\}\right\rangle=\exp \left\{\frac{m}{2 a} \sum_{i} \frac{i(M-i)}{M} J_{i}^{2}+\frac{m}{a} \sum_{i<j} \frac{i(M-j)}{M} J_{i} J_{j}\right. \\
\left.+\frac{q_{M}}{M} \sum_{i} i J_{i}+\frac{q_{0}}{M} \sum_{i}(M-i) J_{i}\right\}
\end{array}
$$

Differentiating $(2 . \overline{1} 0)$ twice with respect to $J_{i}$ and setting all $J=0$, we find

$$
\begin{aligned}
\left\langle\boldsymbol{q}_{l}^{j 2}\right\rangle & =\left[\frac{\boldsymbol{q}_{M}}{M} l+\frac{\boldsymbol{q}_{0}}{M}(M-l)\right]^{2}+\frac{m}{a} \frac{l(M-l)}{M} \\
\frac{1}{M} \sum_{l=1}^{M-1}\left\langle\boldsymbol{q}_{l}^{j 2}\right\rangle & =\int_{0}^{1} d x\left[\boldsymbol{p}_{1} x+\boldsymbol{p}_{0}(1-x)\right]^{2}+\frac{m M}{6 a}+O\left(\frac{1}{M}\right) \\
& =2 \int_{0}^{1} d x \beta(x)+\frac{m M}{6 a}+O\left(\frac{1}{M}\right)
\end{aligned}
$$

where in the last line we have used the boundary conditions $\boldsymbol{q}_{0}=\boldsymbol{p}_{0}, \boldsymbol{q}_{M}=\boldsymbol{p}_{1}$. 
Referring to $\left(\overline{2} . \overline{2}^{i}\right)$, we see that we can represent the necessary counter-term as

$$
\hat{g}^{2} \frac{a N}{2 m M} \int d x \beta(x)=\frac{a}{2 m}\left[\frac{N}{2 M^{2}} \sum_{l=1}^{M-1}\left\langle\boldsymbol{q}_{l}^{j 2}\right\rangle-\frac{m N}{12 a}\right]=\sum_{j=1}^{N} \sum_{l=1}^{M-1}\left\langle\hat{g}^{2} \frac{a}{4 m M^{2}} \boldsymbol{q}_{l}^{j 2}\right\rangle-\hat{g}^{2} \frac{N}{24} .
$$

After representing the $1 / M^{2}$ in the summand by local modifications of the ghost action near the point $(l, j)$, the first term can be seen as a sum over all locations of a local world sheet insertion. The last term is precisely of the right structure to be absorbed in a boundary perimeter term (boundary "cosmological constant"). We have already seen in [12] that such a perimeter term is needed to properly include a mass for the scalar field in the worldsheet description, so it is not surprising that in the process of mass renormalization we should be required to adjust its value to make the final answer covariant.

Summarizing, we have found that if we use our new $u v$ cutoff $\delta$, then for $D<6$ the mass shift shows no non-covariant artifacts, and the divergence (for $4 \leq D<6$ ) can be covariantly absorbed in $\mu^{2}$. For $D=6$ there is a finite non-covariant artifact in the mass shift which can be canceled by a worldsheet local counter-term together with an adjustment of the value of the boundary cosmological constant. The remaining ultraviolet divergences are covariant and can be absorbed in $\mu^{2}$.

\section{Wave function renormalization}

Before moving on to the three point vertex we need to analyze wave function renormalization, which though finite for $D<6$ will show $\log$ divergences at $D=6$, which will contribute to the renormalization of the coupling $\hat{g}$. For this it is convenient to work in energy space by defining

$$
T(E)=\sum_{N=1}^{\infty} e^{a E N} T_{N}
$$

where $T_{N}$ is the amplitude for evolution through $N$ time steps. Then the free gluon propagator is simply

$$
\Delta_{0}\left(p^{2}\right)=\sum_{N=1}^{\infty} \exp \left\{(a E-\lambda) N-N a \frac{\left(\boldsymbol{p}_{1}-\boldsymbol{p}_{0}\right)^{2}+\mu^{2}}{2 m M}\right\}=\frac{1}{e^{a\left(p^{2}+\mu^{2}\right) / 2 m M+\lambda}-1}
$$

where we have defined the off-shell four momentum $p=\left(\boldsymbol{p}, p^{+}, p^{-}\right)=\left(\boldsymbol{p}_{1}-\boldsymbol{p}_{0}, m M, E\right)$. We have also included a boundary cosmological constant $\lambda=O\left(\hat{g}^{2}\right)$ which, as we have seen, will be necessary to cancel non-covariant artifacts in loop diagrams.

Now we include up to one loop corrections to the full propagator

$$
\begin{aligned}
& \Delta\left(p^{2}\right)=\Delta_{0}\left(p^{2}\right)\left\{1+\Delta_{0}\left(p^{2}\right)\left(\Pi\left(p^{2}\right)+\Pi_{\text {С.T. }}\right)+O\left(\hat{g}^{4}\right)\right\} \\
& \Pi\left(p^{2}\right)=\frac{\hat{g}^{2}}{M} \sum_{k, l} \frac{1}{l(M-l)} \frac{1}{(k M / l(M-l)+m \delta / a)^{2}} \exp \left\{-\frac{k a}{2 m} \frac{p^{2}+\mu^{2}}{M}-2 k \lambda\right\} \\
& \exp \left\{-\frac{k a \mu^{2}}{2 m}\left(\frac{M}{l(M-l)}-\frac{1}{M}\right)-\frac{k a \delta}{2 M}\left[\frac{\left(l \boldsymbol{p}_{1}-(M-l) \boldsymbol{p}_{0}\right)^{2}}{k a M+m l(M-l) \delta}\right]\right\} \text {. }
\end{aligned}
$$


Since both the mass shift $\delta \mu^{2}$ and $\lambda$ are of order $O\left(\hat{g}^{2}\right)$, we may, to this order, replace $\mu$ by its physical value and drop $\lambda$ in the expression for $\Pi$. We express $\Delta_{0}$ in terms of the physical mass $\mu_{p h}^{2}=\mu^{2}+\delta \mu^{2}$, expand it to first order in $\hat{g}^{2}$, and plug into (3.4)

$$
\begin{aligned}
& \Delta_{0}\left(p^{2}\right)=\hat{\Delta}_{0}\left(p^{2}\right)+\hat{\Delta}_{0}\left(p^{2}\right)^{2}\left(e^{a\left(p^{2}+\mu_{p h}^{2}\right) / 2 m M}\left(\frac{a \delta \mu^{2}}{2 m M}-\lambda\right)+\Pi\left(p^{2}\right)+\Pi_{C . T .}\right)+O\left(\hat{g}^{4}\right) \\
& \hat{\Delta}_{0}\left(p^{2}\right)=\frac{1}{e^{a\left(p^{2}+\mu_{p h}^{2}\right) / 2 m M}-1}
\end{aligned}
$$

The physical mass is of course defined by the requirement

$$
\Pi\left(-\mu_{p h}^{2}\right)+\Pi_{C . T .}+\left(\frac{a \delta \mu^{2}}{2 m M}-\lambda\right)+O\left(\hat{g}^{4}\right)=0,
$$

which reproduces the covariant mass shift already discussed. When this condition is met the $p^{2} \rightarrow-m_{p h}^{2}$ limit of the quantity in square brackets exists and is equal to the wave function renormalization constant $Z$ :

$$
\begin{aligned}
Z & =\lim _{p^{2} \rightarrow-m_{p h}^{2}}\left[1+\hat{\Delta}_{0}\left(p^{2}\right)\left(p^{2}+\mu_{p h}^{2}\right)\left\{\frac{a}{2 m M}\left(\frac{a \delta \mu^{2}}{2 m M}-\lambda\right)+\Pi^{\prime}\left(-\mu_{p h}^{2}\right)\right\}\right] \\
& =1+\frac{a \delta \mu^{2}}{2 m M}-\lambda+\frac{2 m M}{a} \Pi^{\prime}\left(-\mu_{p h}^{2}\right)
\end{aligned}
$$

The discussion so far has retained both the worldsheet lattice cutoffs and the $u v$ cutoff $\delta$. Now we simplify the expression for $Z$ by taking the worldsheet continuum limit $M \rightarrow \infty$ holding $\delta$ fixed:

$$
\begin{gathered}
Z \underset{M \rightarrow \infty}{\rightarrow} 1-\lambda-\hat{g}^{2} \int_{0}^{1} d x x(1-x) \int_{0}^{\infty} \frac{T d T}{(T+\delta)^{2}} \exp \left\{-\alpha(x) T-\frac{\delta T}{T+\delta} \beta(x)\right\} \\
\underset{\delta \rightarrow 0}{\sim} 1+\frac{\hat{g}^{2}}{6} \ln \left(\delta \mu^{2}\right)-\lambda+\hat{g}^{2} \int_{0}^{1} d x x(1-x)\left(1-\Gamma^{\prime}(1)+\ln \left(\alpha(x) / \mu^{2}\right)\right)
\end{gathered}
$$

where in the last line we see the log divergence as $\delta \rightarrow 0$. From our earlier considerations, we know that the value of $\lambda$ to this order should be $\lambda=\hat{g}^{2} / 24$.

\section{The triangle graph and coupling renormalization}

We first evaluate the 1PIR one loop correction to the cubic vertex shown in Fig. '22, which is finite for $d<4$. We do the calculation in the presence of the $u v$ cutoff $\delta$ introduced in the last section. There are two relevant kinematic configurations in which the spatial location of the loop $l$ is in the range $0<l<M_{1}$ and $M_{1}<l<M$ respectively. We work out the first case, depicted in the figure, in great detail and then briefly discuss the second case. 

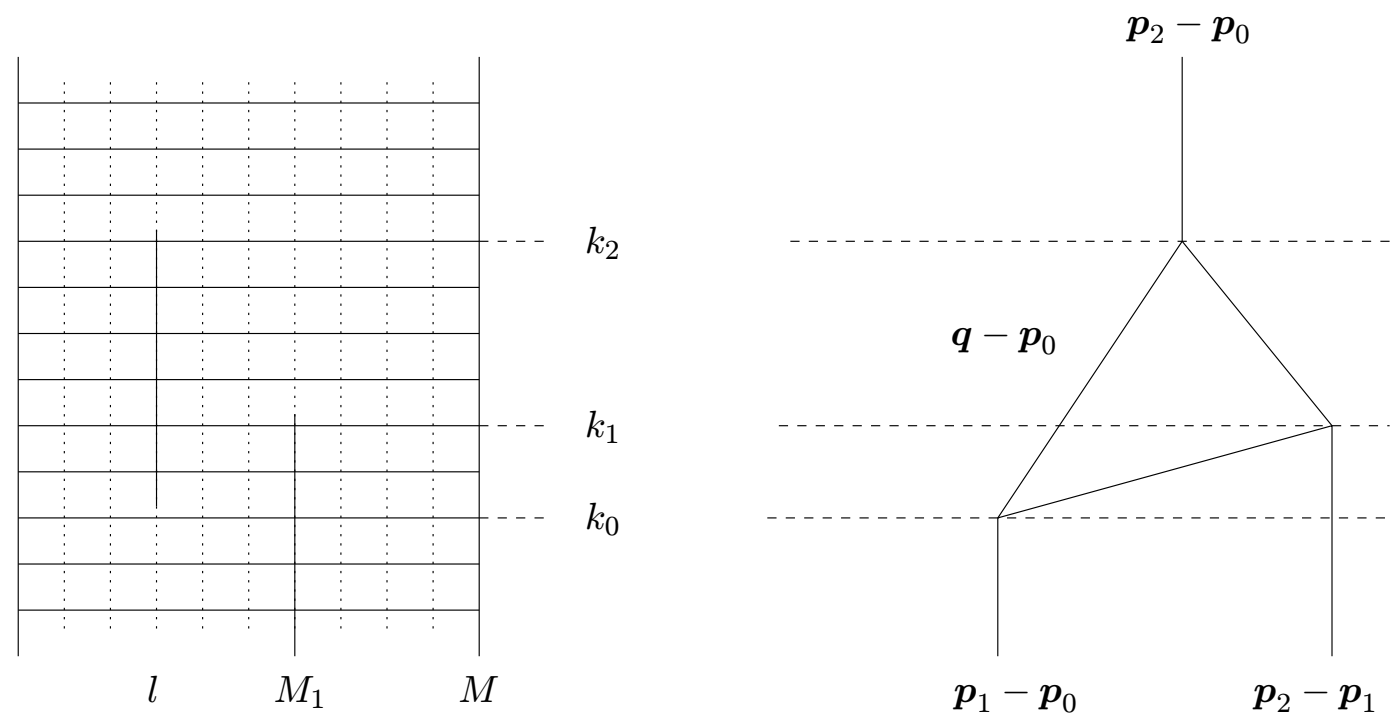

Figure 2: Worldsheet for the triangle diagram shown on the right.

We find for $0<l<M_{1}$, introducing an off shell energy $E=E_{1}+E_{2}$

$$
\begin{aligned}
G_{3}^{1}= & \left(\frac{a}{2 \pi m}\right)^{d / 2} \frac{\hat{g}^{3}}{M} \sum \int d \boldsymbol{q} \frac{1}{l\left(M_{1}-l\right)(M-l)} \exp \left\{-\frac{\left(N-k_{2}\right) a}{2 m}\left[\frac{\left(\boldsymbol{p}_{2}-\boldsymbol{p}_{0}\right)^{2}+\mu^{2}}{M}\right]\right\} \\
& \exp \left\{-\frac{\left(k_{2}-k_{1}\right) a}{2 m}\left[\frac{\left(\boldsymbol{q}-\boldsymbol{p}_{0}\right)^{2}+\mu^{2}}{l}+\frac{\left(\boldsymbol{p}_{2}-\boldsymbol{q}\right)^{2}+\mu^{2}}{M-l}\right]-\frac{\delta}{2} \boldsymbol{q}^{2}\right\} \\
& \exp \left\{-\frac{\left(k_{1}-k_{0}\right) a}{2 m}\left[\frac{\left(\boldsymbol{q}-\boldsymbol{p}_{0}\right)^{2}+\mu^{2}}{l}+\frac{\left(\boldsymbol{p}_{1}-\boldsymbol{q}\right)^{2}+\mu^{2}}{M_{1}-l}+\frac{\left(\boldsymbol{p}_{2}-\boldsymbol{p}_{1}\right)^{2}+\mu^{2}}{M-M_{1}}\right]\right\} \\
& \exp \left\{-\frac{k_{0} a}{2 m}\left[\frac{\left(\boldsymbol{p}_{1}-\boldsymbol{p}_{0}\right)^{2}+\mu^{2}}{M_{1}}+\frac{\left(\boldsymbol{p}_{2}-\boldsymbol{p}_{1}\right)^{2}+\mu^{2}}{M-M_{1}}\right]+a N E-a k_{0}^{\prime} E_{2}\right\} \\
= & \left(\frac{a}{m}\right)^{d / 2} \frac{\hat{g}^{3}}{M} \sum \frac{1}{l\left(M_{1}-l\right)(M-l)} \frac{\Delta_{0}\left(Q_{1}^{2}\right) \Delta_{0}\left(Q_{2}^{2}\right) \Delta_{0}\left(Q_{3}^{2}\right)}{\left(T_{1}+T_{2}+T_{3}+\delta\right)^{d / 2}} \exp \left\{-\frac{\mu^{2}}{2}\left(T_{1}+T_{2}+T_{3}\right)\right\} \\
& \exp \left\{-\frac{T_{1} T_{3} Q_{1}^{2}+T_{1} T_{2} Q_{2}^{2}+T_{2} T_{3} Q_{3}^{2}}{2\left(T_{1}+T_{2}+T_{3}\right)}-\frac{\delta}{2}\left[\frac{\left(\boldsymbol{p}_{0} T_{3}+\boldsymbol{p}_{1} T_{1}+\boldsymbol{p}_{2} T_{2}\right)^{2}}{\left(T_{1}+T_{2}+T_{3}+\delta\right)\left(T_{1}+T_{2}+T_{3}\right)}\right]\right\}(4.2)
\end{aligned}
$$

In this formula we have introduced the $T_{i}$ defined by

$$
T_{1}=\frac{a}{m} \frac{k_{1}-k_{0}}{M_{1}-l}, \quad T_{2}=\frac{a}{m} \frac{k_{2}-k_{1}}{M-l}, \quad T_{3}=\frac{a}{m} \frac{k_{2}-k_{0}}{l} .
$$

the integers $N_{1}=k_{1}-k_{0}, N_{2}=k_{2}-k_{1}$ range independently over the positive integers. We have also introduced the off shell $d+2$ momenta

$$
\begin{aligned}
& Q_{1}=\left(\boldsymbol{p}_{1}-\boldsymbol{p}_{0}, m M_{1}, E-E_{2}\right), \\
& Q_{2}=\left(\boldsymbol{p}_{2}-\boldsymbol{p}_{1}, m\left(M-M_{1}\right), E_{2}\right), \\
& Q_{3}=\left(\boldsymbol{p}_{2}-\boldsymbol{p}_{0}, m M, E\right) .
\end{aligned}
$$

The sums over $N-k_{2}, k_{0}$, and $k_{1}-k_{0}^{\prime}$ just produce the external leg propagators $\Delta_{0}\left(Q_{i}^{2}\right)$. The integer $l$ takes all values $0<l<M_{1}$. 
In the worldsheet continuum limit the sums over $N_{1}, N_{2}, l$ will be replaced by integrals over $T_{1}, T_{2}, T_{3}$, so we shall need the Jacobian

$$
\frac{\partial\left(T_{1}, T_{2}, T_{3}\right)}{\partial\left(N_{1}, N_{2}, l\right)}=\left(\frac{a}{m}\right)^{2} \frac{T_{1}+T_{2}+T_{3}}{l(M-l)\left(M_{1}-l\right)}
$$

The full range $0<T_{i}<\infty$ is covered in the continuum limit when the result $G_{3}^{2}$ of the calculation with $M_{1}<l<M$ is combined with the one discussed above. We then obtain for the worldsheet continuum limit with fixed $\delta$ of the sum of both diagrams:

$$
\begin{gathered}
G_{3}^{1}+G_{3}^{2}=\Delta_{0}\left(Q_{1}^{2}\right) \Delta_{0}\left(Q_{2}^{2}\right) \Delta_{0}\left(Q_{3}^{2}\right) \frac{\hat{g}^{3}}{M}\left(\frac{a}{m}\right)^{(d-4) / 2} \int_{0}^{\infty} \frac{d T_{1} d T_{2} d T_{3}}{T_{1}+T_{2}+T_{3}}\left(T_{1}+T_{2}+T_{3}+\delta\right)^{-d / 2} \\
\quad \exp \left\{-\frac{\mu^{2}}{2}\left(T_{1}+T_{2}+T_{3}\right)-\frac{T_{1} T_{3} Q_{1}^{2}+T_{1} T_{2} Q_{2}^{2}+T_{2} T_{3} Q_{3}^{2}}{2\left(T_{1}+T_{2}+T_{3}\right)}\right\} \\
\exp \left\{-\frac{\delta}{2}\left[\frac{\left(\boldsymbol{p}_{0} T_{3}+\boldsymbol{p}_{1} T_{1}+\boldsymbol{p}_{2} T_{2}\right)^{2}}{\left(T_{1}+T_{2}+T_{3}+\delta\right)\left(T_{1}+T_{2}+T_{3}\right)}\right]\right\}
\end{gathered}
$$

We see explicitly that the $\delta \rightarrow 0$ limit is finite for $d<4$. For $d=4$ (6 space-time dimensions), the integral is only logarithmically divergent in this limit so it is safe to set $\delta=0$ in the exponent for all $d \leq 4$. Adding the tree contribution, we see that up to one loop the 1PIR three vertex is as $\delta \rightarrow 0$ just the tree value times the factor

$$
\begin{gathered}
\frac{1}{Z_{1}}=1+\hat{g}^{2}\left(\frac{a}{m}\right)^{(d-4) / 2} \int_{0}^{\infty} \frac{d T_{1} d T_{2} d T_{3}}{\left(T_{1}+T_{2}+T_{3}\right)^{1+d / 2}} \exp \left\{-\frac{\mu^{2}}{2}\left(T_{1}+T_{2}+T_{3}\right)\right\} \\
\exp \left\{-\frac{T_{1} T_{3} Q_{1}^{2}+T_{1} T_{2} Q_{2}^{2}+T_{2} T_{3} Q_{3}^{2}}{2\left(T_{1}+T_{2}+T_{3}\right)}\right\}
\end{gathered}
$$

for $d<4$. For $d=4$, we extract the log divergence by breaking the integration domain into a region with $T_{1}+T_{2}+T_{3}>\epsilon$ for which we may set $\delta=0$ and a region $T_{1}+T_{2}+T_{3}<\epsilon$ for which we may drop the exponent and then evaluate it explicitly:

$$
\int_{T_{1}+T_{2}+T_{3}<\epsilon} \frac{d T_{1} d T_{2} d T_{3}}{\left(T_{1}+T_{2}+T_{3}\right)\left(T_{1}+T_{2}+T_{3}+\delta\right)^{2}}=\frac{1}{2} \int_{0}^{\epsilon} \frac{T d T}{(T+\delta)^{2}} \sim \frac{1}{2}\left(\ln \frac{\epsilon}{\delta}-1\right)
$$

Then the modification factor for $d=4$ can be written

$$
\begin{aligned}
\frac{1}{Z_{1}} \sim 1+ & \frac{\hat{g}^{2}}{2}\left(\ln \frac{\epsilon}{\delta}-1\right)+\hat{g}^{2} \int_{T_{1}+T_{2}+T_{3}>\epsilon} \frac{d T_{1} d T_{2} d T_{3}}{\left(T_{1}+T_{2}+T_{3}\right)^{3}} \\
& \exp \left\{-\frac{\mu^{2}}{2}\left(T_{1}+T_{2}+T_{3}\right)-\frac{T_{1} T_{3} Q_{1}^{2}+T_{1} T_{2} Q_{2}^{2}+T_{2} T_{3} Q_{3}^{2}}{2\left(T_{1}+T_{2}+T_{3}\right)}\right\}
\end{aligned}
$$

Incorporating the wave function renormalization factors $Z^{3 / 2}$, we see that the divergence can be absorbed in a renormalized coupling

$$
\hat{g}_{r}=\hat{g}\left(1+\frac{\hat{g}^{2}}{2} \ln \frac{1}{\mu^{2} \delta}+\frac{3}{2} \frac{\hat{g}^{2}}{6} \ln \mu^{2} \delta\right)=\hat{g}\left(1+\frac{\hat{g}^{2}}{4} \ln \frac{1}{\mu^{2} \delta}\right)
$$

Recall that $\hat{g}^{2}=g^{2} / 64 \pi^{3}$, where $g$ is the conventionally defined coupling. In terms of it, the renormalization reads

$$
g_{r}=g\left(1+\frac{g^{2}}{256 \pi^{3}} \ln \frac{1}{\mu^{2} \delta}\right)
$$


and the Callan-Symanzik beta function is

$$
\beta\left(g_{r}\right) \equiv \mu \frac{d g_{r}}{d \mu}=-\frac{g_{r}^{3}}{128 \pi^{3}}+O\left(g_{r}^{5}\right)
$$

To compare to the standard result, remember that this result is strictly the $N_{c} \rightarrow \infty$ limit, and $g$ is $\sqrt{N_{c}}$ times the conventional coupling. At finite $N_{c}$ one can decompose $\Phi$ into adjoint and singlet components. Then one finds different renormalizations for the $A d j^{3}$,

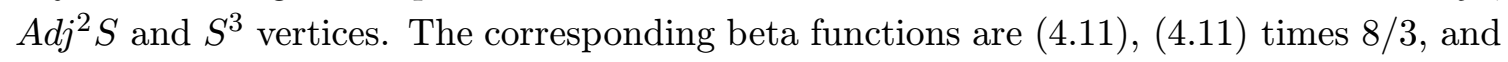
(4) $\left.411_{1}\right)$ times 6 , respectively. Then the $N_{c}=1$ beta function is to be compared to the one for the $S^{3}$ vertex. The field $\Phi$ for the case $N_{c}=1$ is just a single hermitian scalar field. But with our definition the, cubic term goes to $g \Phi^{3} / 3$ instead of the customary $g \Phi^{3} / 3$ !; after taking this difference into account, which means multiplying our beta function by $1 / 4$, we get the known result for $N_{c}=1$, with $-3 / 256 \pi^{3}$ multiplying the customary coupling cubed.

\section{Conclusion}

The worldsheet "template" for summing planar diagrams has been set up for a whole range of interesting theories, including QCD and supersymmetric gauge theories. In this talk I have shown in detail how the field theoretic renormalization program plays out on the worldsheet for theories with scalar cubic couplings through one loop. We have given in this case a local worldsheet description of the counter-terms necessary for one loop Lorentz invariance for space-time dimensions $D \leq 6$. More generally it is hoped that the principle of worldsheet locality will assist the renormalization program for gauge theories in lightcone gauge. Because field theoretic locality is not manifest in this gauge it is of no direct use in restricting counter-terms. The new worldsheet locality, if it survives the regulation of $u v$ divergences, will be manifest and will therefore provide a new principle for classifying counter-terms. The main obstacle still to be overcome is the worldsheet regulation of the $p^{+}=0$ singularities that occur in gauge theories in light-cone gauge.

The eventual goal of the worldsheet description of field theory is to bring the powerful techniques of string theory to bear on the problem of quark confinement in QCD. ${ }^{2}$ There is a remote chance that it will enable a completely analytic understanding of this important problem. But even if this is not possible, a numerical attack on the worldsheet formulation of the problem may offer insights complementary to those provided by lattice gauge theory. In particular, the two-dimensionality of the worldsheet lattice promises to bring new efficiencies to numerical spectrum calculations, perhaps allowing a closer approach to the continuum answers.

Acknowledgments: I am grateful to K. Bardakci and S. Glazek for valuable discussions. This research was supported in part by the Department of Energy under Grant No. DEFG02-97ER-41029.

\footnotetext{
${ }^{2}$ This goal is of course shared by practitioners of the AdS/CFT correspondence [13-16].
} 


\section{References}

[1] K. Bardakci and C. B. Thorn, Nucl. Phys. B626 (2002) 287, hep-th/0110301.

[2] G. 't Hooft, Nucl. Phys. B72 (1974) 461.

[3] C. B. Thorn, Nucl. Phys. B 637 (2002) 272 [arXiv:hep-th/0203167].

[4] S. Gudmundsson, C. B. Thorn, and T. A. Tran Nucl. Phys. B 649 (2002) 3, [arXiv:hep-th/0209102].

[5] C. B. Thorn, [arXiv:hep-th/0310121].

[6] P. Goddard, J. Goldstone, C. Rebbi, and C. B. Thorn, Nucl. Phys. B56 (1973) 109.

[7] R. Giles and C. B. Thorn, Phys. Rev. D16 (1977) 366; C. B. Thorn, Phys. Lett. 70B (1977) 85; Phys. Rev. D17 (1978) 1073; K. Bering, J. S. Rozowsky and C. B. Thorn, Phys. Rev. D61 (2000) 045007, hep-th/9909141.

[8] K. Bardakci and C. B. Thorn, Nucl. Phys. B 661 (2003) 235, [arXiv:hep-th/0212254].

[9] K. Bardakci and C. B. Thorn, Nucl. Phys. B 652 (2003) 196,[arXiv:hep-th/0206205].

[10] K. Bardakci, "Self consistent field method for planar phi**3 theory," arXiv:hep-th/0308197.

[11] S. D. Glazek, Phys. Rev. D 60 (1999) 105030 [arXiv:hep-th/9904029]; Phys. Rev. D 63 (2001) 116006 [arXiv:hep-th/0012012]; Phys. Rev. D 66 (2002) 016001 [arXiv:hep-th/0204171]; arXiv:hep-th/0307064.

[12] C. B. Thorn and T. A. Tran, arXiv:hep-th/0307203.

[13] J. M. Maldacena, Adv. Theor. Math. Phys. 2 (1998) 231-252, hep-th/9711200.

[14] I. R. Klebanov and M. J. Strassler, JHEP 0008 (2000) 052 [arXiv:hep-th/0007191].

[15] J. Polchinski and M. J. Strassler, arXiv:hep-th/0003136; Phys. Rev. Lett. 88 (2002) 031601 [arXiv:hep-th/0109174]; JHEP 0305 (2003) 012 [arXiv:hep-th/0209211].

[16] D. Berenstein, J. M. Maldacena and H. Nastase, JHEP 0204 (2002) 013 [arXiv:hep-th/0202021]. 